\title{
Dinámicas y prácticas de cuidado en la pobreza, la vejez y la discapacidad: La familia Vargas ${ }^{1}$
}

\author{
Alba Lucero López Díaz² \\ Vilma Florisa Velázquez ${ }^{3}$ \\ Martha Lucia Alzate Posada4 \\ José Carlos Bom-Mehyl ${ }^{5}$
}

doi:10.11144/Javeriana.ie19-1.dpcp

Cómo citar: López Díaz AL, Velázquez VF, Alzate Posada ML, Bom-Mehyl JC. Dinámicas y prácticas de cuidado en la pobreza, la vejez y la discapacidad: La familia Vargas Imagen Desarr. 2017;19(1): 139-151. http://dx.doi.org/10.11144/Javeriana.ie19-1.dpcp

1. Articulo original de investigacion.Fecha de recibido: 18 de junio de 2015. Fecha de aceptacion: 13 de enero de 2016.

2. Profesora Asociada - Facultad de Enfermería de la Universidad Nacional de Colombia, Doctora y Magíster en Enfermería de la Universidad de Sao Paulo/Brasil. Líder del Grupo de Investigación en Cuidado Cultural de la Salud. Correo electrónico: allopezdi@ unal.edu.co

3. Profesora Asociada - Facultad de Enfermeria de la Universidad Nacional de Colombia, MSc en Enfermería y Educación de Adultos. Investigadora del Grupo de Investigación en Cuidado Cultural de la Salud. Correo electrónico: vvelasquez@unal.edu.co

4. Profesora Asociada - Facultad de Enfermería de la Universidad Nacional de Colombia, MSc Administración en Salud, PhD Salud Pública de la Universidad de Sao Paulo - Brasil. Investigadora del Grupo de Investigación en Cuidado Cultural de la Salud. Correo electrónico: mlalzatep@unal.edu.co

5. Oralista. PhD. en Historia Social. Profesor Titular de la Universidade de Sao Paulo. Director del Núcleo de Historia Oral (NEHO) de la Universidad de Sao Paulo - Brasil. Correo electrónico: jcarlosbm@hotmail.com 


\section{Resumen}

Introducción: Para el profesional de salud que busca ofrecer un cuidado congruente con los valores, creencias, prácticas y necesidades sentidas es un desafío conocer la heterogeneidad y diversidad cultural de un grupo de población que envejece de forma acelerada en el mundo. Objetivo: Este artículo describe las dinámicas y las prácticas de cuidado en salud de una familia en condición de pobreza que vive con una persona anciana en situación de discapacidad en la ciudad de Bogotá (Colombia). Método: Estudio cualitativo desarrollado con base en la historia oral de vida, tejido con la participación de tres integrantes de la familia Vargas. Las entrevistas en profundidad suman 894 minutos de grabación. La recolección, procesamiento y análisis de datos se efectuó siguiendo el procedimiento propuesto por el profesor e investigador brasilero José Carlos Sebe Bom Meihy. Resultados: Dos grandes temas emergieron: La familia Vargas: entre lo glocal y lo líquido, y Los Vargas: entre la sobrevivencia y la lucha por la dignidad. Conclusiones: El cuidado ocurre como una expresión de solidaridad familiar en una dinámica ambivalente y tensa, con cargas económicas, emocionales y de deterioro en la calidad de vida para las personas que lo asumen. La familia soporta en la esfera privada la dura carga de cuidar, acumulando muchas más desventajas sociales quedando surcados en un círculo de humillación e indignidad.

Palabras clave: familia; anciano; personas con discapacidad: cultura; enfermeria transcultural

\section{Dynamics and care practices in poverty, old age and disability: The Vargas family}

\section{Abstract}

Introduction: For health professionals that seek to provide care which aligns with the values, beliefs, practices and perceived need, it is a challenge to know the heterogeneity and cultural diversity of a population group which is rapidly aging. Objective: This article describes the dynamics and health care practices of a family in poverty living with an elderly person with disabilities in the city of Bogotá (Colombia). Method: A qualitative study was developed based on the oral history of life, constructed with the participation of three members of the Vargas family. In-depth interviews total 894 minutes of recording. The collection, processing and data analysis was carried out following the procedure proposed by the Brazilian professor and researcher José Carlos Sebe Bom Meihy. Results: Two major themes emerged: The Vargas family: Between glocal and liquid and The Vargas: between survival and struggle for dignity. Conclusions: The care occurs as an expression of family solidarity in an ambivalent and tense dynamic, with economic and emotional loads which bring deterioration in the quality of life for those who take them. The family supports in the private sphere the heavy burden of care, accumulating many more social disadvantages and in turn end up trapped in a circle of humiliation and indignity.

Keywords: family; old man; people with disabilities: culture; transcultural nursing 


\section{Dinâmicas e práticas de cuidado na pobreza, a velhice e a deficiência: a família Vargas}

\section{Resumo}

Introdução: Para o profissional de saúde que visa proporcionar um cuidado congruente com os valores, crenças, práticas e necessidades sentidas é um desafio conhecer a heterogeneidade e diversidade cultural de uma turma de população que envelhece de forma acelerada no mundo. Objetivo: Este artigo descreve as dinâmicas e práticas de cuidado em saúde de uma família em situação de pobreza que vive com uma pessoa idosa com deficiência, na cidade de Bogotá (Colômbia). Método: Estudo qualitativo desenvolvido com base na história oral de vida, entretecido com a participação de três integrantes da família Vargas. As entrevistas em profundidade totalizam $894 \mathrm{mi}$ nutos de gravação. A coleta, processamento e analise de dados foi efetuado segundo o procedimento proposto pelo professor e pesquisador brasileiro José Carlos Sebe Bom Meihy. Resultados: Dois grandes temas emergiram: a família Vargas: entre o glocal e o líquido e Os Vargas: entre a sobrevivência e a luta pela dignidade. Conclusões: O cuidado ocorre como expressão de solidariedade familiar sob uma dinâmica ambivalente e tensa, com cargas econômicas, emocionais e de deterioro na qualidade de vida para as pessoas que o assumem. A família suporta, na esfera privada, o pesado fardo de cuidar, acumulando muitas mais desvantagens sociais saindo franzidos em um círculo de humilhação e indignidade.

Palavras-chave: família; idoso; pessoas deficientes: cultura; enfermagem transcultural 


\section{Introducción}

En la actualidad los temas de vejez o "adulto mayor", pobreza y discapacidad cobran fuerza en las agendas politicas de los gobiernos a partir del incremento del envejecimiento poblacional y la longevidad, del aumento de la población con situaciones de discapacidad y pobreza; una realidad de orden mundial, regional, nacional y local, y una población caracterizada por nuevos perfiles demográficos, diversidad sociocultural, nuevas modalidades de migraciones y desplazamiento, así como también, un acelerado y complejo envejecimiento de la población.

Según el informe conciso de las Naciones Unidas sobre la situación demográfica del mundo, la población en condición de vejez ha aumentado a un $12 \%$ en el 2014 y se espera que para el 2050 este porcentaje llegue al $21 \%$, siendo mayor en las mujeres (del citado porcentaje unos 392 millones serán personas mayores de 80 años). Este incremento trae consigo múltiples retos de orden político, económico, social y de prestación de servicios públicos como la salud, seguridad y protección social, dado que cada vez más, esta población es vulnerable a eventos como la pobreza, enfermedad, discapacidad, abandono, desempleo, con los consecuentes cambios en la dinámica familiar e incremento en el índice de dependencia (1).

La OMS informa que la prevalencia de discapacidad está en aumento. Más de mil millones de personas en todo el mundo tienen alguna forma de discapacidad, de las cuales más de 200 millones padecen dificultades considerables en su funcionamiento y por ende algún grado de dependencia. Al aumentar el envejecimiento poblacional, el riesgo de discapacidad aumenta en los adultos mayores, se presenta un incremento en la incidencia y prevalencia de las enfermedades crónico degenerativas. "En todo el mundo, las personas con discapacidad tienen peores resultados sanitarios, peores resultados académicos, una menor participación económica y unas tasas de pobreza más altas que las personas sin discapacidad"(2); debido en parte, a las múltiples barreras de acceso a servicios públicos, en particular la salud, la educación, el empleo, el transporte o la información. Esas dificultades se exacerban en las comunidades menos favorecidas como lo son los adultos mayores, persisten y se traducen en desigualdades sociales que se incrementan en la vejez, manifestándose de diferentes maneras en cada género y repercutiendo en el estado de salud de las personas adultas mayores $(2,3)$.

Desde la II Asamblea Mundial sobre Envejecimiento (Naciones Unidas, 2002) y la Organización Mundial de la Salud (2002) en su política de envejecimiento activo, se expresa la necesidad de adoptar politicas, programas e investigaciones que aborden la pobreza y la discapacidad en la vejez por corresponder a factores de gran amenaza para el bienestar y calidad de vida de las personas, exponiéndolas, cada vez más, a la exclusión social, al maltrato, a la fragilidad de la salud, la pérdida de la autonomía y mayor vulnerabilidad frente a situaciones de emergencia $(4,5)$.

En América Latina la pobreza, la discapacidad y la vejez están estrechamente relacionadas con una condición de vulnerabilidad y de pérdida de bienestar que conlleva a mayor empobrecimiento. En Colombia la población mayor de 60 años para el 2012 superó un 10\% y se espera que para el 
2015 sea superior al 20\% (DANE, 2010) más concentrada en las ciudades y con una población rural cada vez más envejecida (6). Según diferentes boletines del DANE y el análisis de la situación del envejecimiento demográfico en Colombia 1951-2020, existían 4'964.793 personas mayores de 60 años en el 2013, que corresponden al 10,5\% de la población. De ese total, 650.000 sobrepasan los 80 años. De ellos, el 52\% de los adultos mayores se encuentran en condición de pobreza (6). El índice de envejecimiento (relación entre mayores de 60 años y los menores de 15 años) demuestra que desde 1951 ha habido un aumento sostenido, al pasar de 12 a 41,5 personas mayores de 60 años por cada 100 jóvenes. Eso permite proyectar que antes de cinco años la proporción será de 50 adultos mayores por cada 100 jóvenes en nuestro medio $(6,7)$.

Igualmente, las personas ancianas y con discapacidad envejecen en condiciones de pobreza como resultado de una vida de privaciones, de un escaso acceso al sistema de salud, a limitaciones en las oportunidades de empleo y escasas posibilidades para recibir una pensión. La familia asume ese impacto económico y social, mientras que el gobierno no toma un papel decidido para asegurar un envejecimiento con seguridad y dignidad.

Abordar el fenómeno de la vejez, la discapacidad y la pobreza desde la historia oral tiene como motivación la oportunidad de "conocer la propia visión que los sectores populares tienen de sus vidas y del mundo a su alrededor" es abrir las posibilidades de conocer la cotidianidad, de como las familias viven esos fenómenos permeados por la forma en que cada integrante de la familia significa lo vivido, lo que está presente y sobre ello proyectan el futuro (8). Al indagar el fenómeno desde esta perspectiva se puede conocer como la realidad política, social, económica, cultural e individual impacta sobre cada sujeto, y al mismo tiempo, sobre el núcleo familiar, con miras a determinar acciones específicas que pueden ser realizadas desde enfermería con perspectiva cultural y proyección, para que estas sean también asumidas por cualquier profesional de la salud previamente capacitado.

Este abordaje permite, por un lado, dar voz a las personas que integran una familia excluida y silenciada social y culturalmente, y por otro, propicia una reflexión profunda sobre el drama ético de una sociedad moderna que privilegia la concentración de la riqueza, la competitividad y el consumo, dejando en desventaja a un grupo de la población que ve disminuida su capacidad de afrontar el peso social que cae sobre ellos. Incita a la construcción de relaciones más sólidas en contraposición al mundo líquido en una perspectiva glocal es decir una mezcla entre lo global y lo local. En japonés deriva de dochaku "el que vive en su propia tierra" que tanto en el agro como en marketing, es adaptar una visión global a las condiciones locales para impulsar desarrollos de distinta indole (9). Algunas reflexiones sobre la glocalización y salud se pueden leer en López L. (10).

Lo líquido acuñado por Zygmunt Bauman muestra una figura del cambio y de la transitoriedad: "los sólidos conservan su forma y persisten en el tiempo: duran, mientras que los líquidos son informes y se transforman constantemente: fluyen. Como la desregulación, la flexibilización o la liberalización de los mercados" (11). Así, el tránsito de una modernidad "sólida" 
(estable, repetitiva) a una "líquida" (flexible, voluble) en la que los modelos y estructuras sociales ya no perduran lo suficiente como para enraizarse y gobernar las costumbres de los ciudadanos. El amor líquido, entonces, tiende a mostrar una tendencia de las relaciones cada vez más fugaces, superficiales, etéreas y con menor compromiso.

Para Robertson, la preocupación está en ir más allá de la idea de globalización como opuesta a la localización. La glocalización, implica de manera creciente la creación e incorporación de la localidad, procesos que a su vez, configuran ampliamente la comprensión del mundo como un todo. Y al mismo tiempo, el énfasis sobre la condición global —esto es, en la globalidad- nos empuja a que nuestro análisis e interpretación del mundo contemporáneo sean a la vez espacial, temporal, geográfico e histórico (12).

Bajo las anteriores consideraciones y teniendo en cuenta el objetivo central de esta investigación que fue describir las dinámicas y las prácticas de cuidado en salud de una familia en condición de pobreza, que vive con una persona anciana con discapacidad en la ciudad de Bogotá (Colombia), a continuación se describen los resultados más relevantes que deben ser tenidos en cuenta por parte de los profesionales de la salud quienes tienen una responsabilidad social, donde la salud es parte de la dignidad humana y las políticas públicas han de estar más acordes con el contexto cultural de las personas, la familia y la sociedad moderna, y su aplicación debe dar respuestas más acertadas y eficaces.

\section{Método}

Este estudio cualitativo basado en historia oral de vida se llevó a cabo siguiendo la propuesta metodológica de Meihy: se establecieron la colonia, la red de colaboradores y la entrevista, para llegar a la confección del documento y la conferencia (8). Según el citado autor, la historia oral puede darse en tres modalidades: historia oral de vida, historia oral temática y tradición oral. La historia oral de vida, elegida para este estudio, se entiende como "el conjunto de la narrativa de la experiencia de vida de una persona" y en nuestro caso se refiere al tejido de las narrativas individuales al interior de la familia Vargas (8).Luego de que los participantes, que en adelante se llamarán colaboradores, firmaron el consentimiento informado se practicaron las entrevistas a profundidad, y a medida que se iban trascribiendo eran analizadas una a una, para luego proceder a la confección de la historia oral, que fue dada a conocer en la fase de la conferencia de la historia individual de forma completa y fue aceptada la versión final. A cada uno de ellos se le entregó copia de la historia completa. En la presentación de resultados se utilizan apartes de la versión final. Los nombres y apellidos utilizados fueron escogidos por los colaboradores.

Igualmente, esta investigación se ajusta a los principios los cuatro principios bioéticos y científicos fundamentales de Beauchamp y Childress: beneficencia no maleficencia, respeto a la autonomía, libertad y justicia. Se considera investigación sin riesgo dado que las técnicas y métodos de investigación no requieren de intervención o modificación intencionada de las 
variables biológicas, fisiológicas, psicológicas o sociales de los individuos colaboradores del el estudio, según el Artículo No. 11 de la Resolución 8430 de 1993 por la cual se establecen las normas científicas, técnicas y administrativas para la investigación en salud (13).

\section{Muestra}

De acuerdo con Meihy, la colonia parte de la comunidad de destino que a su vez se define como el fragmento representativo de esa comunidad (14). Se tomó como colonia las familias con las que el grupo de investigación había realizado estudios etnográficos anteriores. Así, la colonia partió de 60 familias en situación de pobreza que tienen entre sus integrantes personas ancianas dependientes total o parcialmente para el autocuidado, residentes en dos localidades de la ciudad de Bogotá (Kennedy y San Cristóbal).

Bogotá tiene 20 localidades. La localidad $8^{a}$ de Kennedy está ubicada en el suroccidente de Bogotá, en la zona aluvial del valle del río Bogotá. Cuenta con 118 barrios y alberga 14\% (1030.000 habitantes) de la población capitalina, entre quienes $51 \%$ son mujeres, $70 \%$ corresponde al grupo de edad entre 15 y 64 años, y $6 \%$ son personas mayores de 65 años. Su problemática social, demuestra que esta zona se encuentra en los primeros lugares en cuanto a necesidades humanas básicas insatisfechas. Es la segunda localidad receptora de familias desplazadas y la tercera en situación de pobreza por el método de análisis de Necesidades Básicas Insatisfechas (NBI) que equivale a $5.1 \%$ y corresponde al promedio de la ciudad $(5.2 \%)$. Igualmente, es la segunda localidad con el mayor indicie de homicidios al año (15). En la localidad de Kennedy la esperanza de vida es de 74,7 en el caso de los hombres y 79,7 años para las mujeres. El 5,1\%, de la población de la localidad es pobre de acuerdo al NBI. Para el año 2011, Kennedy se encontraba por debajo al promedio Distrital (5,2\%) (16).

La localidad $4^{a}$ de San Cristóbal se encuentra ubicada en los cerros orientales de la ciudad de Bogotá y está compuesta por 172 barrios. Tiene más de 100 años de historia y está marcada por las desigualdades sociales, la mala planificación, amenazada por la naturaleza, agredida y desconocida. Ocupa el segundo lugar en situación de pobreza en Bogotá. Su población asciende a más de 409.777 habitantes, de los cuales 201.075 son mujeres y 199.724 hombres. El 66.5\% de sus habitantes están entre los 15 y 64 años, el 6.1\% son personas mayores de 65 años. Para el 2015 la esperanza de vida al nacer está en 77 años para las mujeres y 70 para los hombres. Predomina el estrato socioeconómico bajo-bajo, bajo y medio bajo. La razón de dependencia es de 52 personas dependientes por cada 100. Con relación a la pobreza, en el 2011 el $29.6 \%$ de sus habitantes son pobres, $30.2 \%$ del total de la población de la localidad padece pobreza por ingreso y el $6.7 \%$ indigencia (17). Con respecto al predominio de población joven en la localidad, el índice de envejecimiento es de 18.32 para hombres y 26.86 para mujeres, existe una proporción superior de mujeres con más de 65 años. El índice de dependencia de la población mayor de 65 años es de 6,95 y 8,98 para hombres y mujeres por cada 100.000 personas en edad económicamente activa. 
Tanto Kennedy como San Cristóbal son zonas con vulnerabilidad social, cultural y ambiental y con mayores carencias de servicios públicos básicos. Están ubicadas entre las tres primeras localidades del Distrito Capital como densamente pobladas, en proceso de urbanización ilegal. Con altos riesgos y amenazas geológicas (deslizamientos, inundaciones, deforestación, entre otros) debido a terrenos empinados, arcillosos, presencia de humedales, ríos y quebradas. Presentan altos riegos para la salud por contaminación del aire, el agua y el ambiente físico y social. Tienen inadecuado cubrimiento de servicios públicos como agua potable, disposición de basuras y residuos sólidos. Población que demanda servicios de salud especiales y en la mayoría de los casos requiere atención permanente por parte de un cuidador, que por lo general es un familiar que se ve obligado a dejar su trabajo, es mujer o también se encuentra en condiciones de salud deficientes, esto crea nuevas condiciones de salud y vida determinadas socialmente y que ameritan de estudios cada vez diversos en temáticas, metodologías y tópicos como la resiliencia, la determinación social, influencias percibidas de los factores contextuales (18).

La red de colaboradores, según Meihy (14) es una subdivisión de la colonia con la que se busca caracterizar de manera racional elementos específicos de la temática que se indaga. Para la conformación de esta red se buscaron características de contraste en aspectos como: género y grado de dependencia para el autocuidado. La red fue constituida por cuatro familias: dos de Kennedy y dos de San Cristóbal.

La familia Vargas, que reside en la Localidad de San Cristóbal, está constituida por el padre, Octavio un señor de 68 años con un grado de dependencia parcial para la realización de las actividades de la vida diaria, sus dos hijas, Esperanza Vargas de 42 años y Yolima Herrera Vargas de 47 años cuidadoras principales y quienes brindan atención de salud en forma permanente al padre.

De acuerdo con Meihy, la entrevista consta de tres momentos: pre-entrevista, entrevista y post-entrevista. Estas fases fueron desarrolladas en el domicilio de los colaboradores. Para el presente reporte se toma únicamente la historia oral de la familia Vargas de uno de los sectores. Las entrevistas se realizaron con tres miembros de la familia: El señor Vargas y sus dos hijas. Las grabaciones sumaron 894 minutos y fueron realizadas de forma múltiple, individual, y ninguno de los integrantes conoció la narrativa del otro. Los tópicos de indagación circularon frente al desarrollo de la vida de cada uno de ellos y lo que había significado la situación de vejez, discapacidad y pobreza en sus vidas.

La confección del documento que es la materialización de lo oral a lo escrito, es un procedimiento complejo donde se toman una serie de cuidados técnicos que muchas veces escapan a la consideración de quien ve el resultado final (14). Allí está: la transcripción, la textualización y la transcreación, o sea la edición. La historia oral de vida de la familia tuvo 72 versiones en total. Las versiones se fueron construyendo a medida que se llevaban a cabo las entrevistas y se iba organizando y profundizando sobre la 
vida de los integrantes y las temáticas enunciadas. Este procesamiento de información implica un análisis inductivo e interpretativo constante de los datos emergentes, que van cobrando sentido en la construcción de la historia.

\section{Resultados y discusión}

Las historias individuales se conectan en una historia familiar con do s temáticas afines que emergen de las narrativas: La familia Vargas: entre lo glocal, lo liquido, y los Vargas: entre la sobrevivencia y la lucha por la dignidad

\section{La familia Vargas: entre lo glocal y lo líquido}

Son evidentes las complejas transformaciones cotidianas, ambientales, económicas y políticas a escala planetaria. Sin embargo, existe una estrecha interdependencia entre lo global y lo local, que se puede sintetizar en la "glocalizaciónérmino acuñado por Robertson que implica de manera creciente la creación e incorporación de la localidad a procesos que, a su vez, configuran ampliamente la comprensión del mundo como un todo (19). En esa medida una historia local como es la de los Vargas resulta de gran interés al intentar comprenderla desde las conexiones glocales con las diferencias, semejanzas e interconexiones que de ella se pueden derivar.

Octavio Vargas, es un hombre de 68 años, bogotano y zapatero de "profesión". Paradójicamente su momento vital es atravesado por una trágica metáfora al ser un "zapatero sin zapatos"; Su oficio por varios años fue ser zapatero y con el tiempo, perdió sus miembros inferiores, con él se ingresa a una realidad que aunque local, puede ser global, porque permite ver cómo la cotidianidad revela una memoria en la que se entrecruzan los factores políticos, económicos, sociales y culturales.

Mi abuelo fue el que me dio el apellido, como si no hubiera tenido papá... la verdad me crie un tiempo con los abuelos, otro con una tía... me mandaron para la Granja del Padre Luna, donde van los niños abandonados. ¡Luego trabajé desde los nueve años con mi papá... siempre trabajé!... El peor error de la vida es casarse... nos duró 25 y tantos años el matrimonio... pienso que fui un buen papá, porque les di todo para vivir: estudio, techo, comida, les di buen ejemplo, no los maltrataba porque golpes, no les daba. Para mí era importante cuidar las muchachas porque para los hombres es más fácil, quería que ellas se organizaran, que alguien las protegiera... por eso me mantuve en el matrimonio hasta el año 1985, pensaba mucho en ellas. Ahora no sé si ellas se dan cuentan de eso porque uno de esas cosas no sabe...

Hoy puedo decir que la vida ha cambiado mucho... ahora sin las piernas... a lo mejor de tanto martillar los zapatos en la rodilla hizo que no circulara bien la sangre en la pierna, pero también me fumaba 36 cigarrillos diarios, y eso también dañó la coagulación, tapó las venas y dañó mis piernitas... solo ahora que estoy así es que pienso en todo eso. 
Durante muchos años no necesité la ayuda de mis hijas, pero después de todo lo ocurrido ellas me han dado la mano... mis nietos abrieron las puertas de la casa y las de su corazón, y me permitieron alojarme aquí, no juzgaron mi pasado, no miraron mi condición física, simplemente extendieron sus brazos y me obligaron a tomar el papel de abuelo que hasta ahora no lo había conocido (Octavio).

Las mujeres que históricamente han ejercido el papel de cuidadoras en esta familia, ahora como hijas, son quienes asumen el apoyo y la tarea como cuidadoras de Octavio. Así se expresa Esperanza, la hija mayor:

La vida con mis padres fue dura por todo lo que les tocaba hacer para conseguir el sustento de sus ocho hijos. Tuve cuatro hijos, los tres primeros fueron niños, la última fue una niña. Me ha tocado rebuscarme la vida como sea... Siempre termina uno siendo la hija que ve por todo... me da nostalgia recordar el día que me fui a trabajar y encontré que habían dejado acá a mi papá... La verdad, no quería tenerlo en esta casa pero tampoco podía botarlo a la calle porque ¡cómo voy a hacer eso! al fin y al cabo es mi papá... (Esperanza).

Yolima dice:

Mi papá era muy brusco con mi mamá, no le ayudaba en nada... Cuando cumplí los 18 años quedé embarazada... ese fue el secreto guardado por muchos años y ¡tristemente! tengo que decir que ese hijito era de él. Tuve seis hijos, el primero fue Juan David, nació sordomudo y murió a los 26 años... El papá de los otros niños nunca nos ayudaba pa' nada pero he salido adelante. He trabajado en restaurantes, en casas de familia, entonces le daba a mis hijos todo lo que necesitaban... nosotros tenemos a mi papá como porque no se vaya pa' la calle, es que también ¿cómo lo vamos a sacar? Mi mamá me dice que no tengo la obligación de tenerlo pero toca hacer la cara dura y llevar las cosas, que más... todo esto es muy duro para nosotras... solo cuando murió mi Juan le conté todo a mi mamá... es por eso que todos los domingos voy a la iglesia Cristiana porque nos enseñan a perdonar las cosas... (Yolima).

Estas narrativas exponen crudas situaciones de orden familiar, social y cultural. Pero ni siquiera, el evidente drama ético familiar de los Vargas responde a las transformaciones sociales imperantes, donde se estimula y aplaude globalmente el individualismo; dice Bauman: “[...] las relaciones suelen ser, quizá, las encarnaciones más comunes, intensas y profundas de la ambivalencia [...]" (19), porque al mismo tiempo se busca el vínculo sin el compromiso donde se "provoca el impulso de estrechar los lazos pero manteniéndolos al mismo tiempo flojos". Esas relaciones que, de acuerdo con Bauman, son impregnadas por el "amor líquido en un tiempo líquido" en la modernidad. Estas tendencias no son las que glocalmente muestran los Vargas, en medio de los conflictos y las historias cotidianas existe la premisa del compromiso 
mutuo, el privilegio de la cristalización de la relación en contraposición a una relación líquida (19).

Entre los Vargas, las contradicciones y dificultades son sorteadas porque la premisa base es que las relaciones no son desechables. Esa lucha cotidiana de los Vargas resulta algo incómoda para "nosotros habitantes del moderno mundo líquido que aborrece todo lo sólido y durable, todo lo que no sirva para el uso instantáneo y que implica esfuerzos sin límite, esa perspectiva supera toda capacidad y voluntad de negociación” (19). Los Vargas que viven en triple condición de vulnerabilidad, son quienes nos muestran que aún existe la posibilidad de las relaciones durables, capaces de negociar y de enfrentar las adversidades. Sin duda, es posible realizar desde distintas miradas otros análisis de la dinámica familiar, pero debemos advertir que en este momento el foco está dado a ver a los Vargas como una construcción de memoria colectiva glocalizada, como una expresión de la resistencia, de las bondades y desventajas que pueden tener las relaciones líquidas en medio de situaciones de pobreza, vejez y discapacidad.

\section{Los Vargas: entre la sobrevivencia y la lucha por la dignidad}

Los Vargas, una familia que vive en condición de pobreza con una persona anciana, evidencia un panorama que crece en el mundo glocalizado. De acuerdo con el Banco Mundial, las personas muy pobres en el mundo (2.800 millones de personas, casi la mitad de la población mundial) viven con menos de US\$2 al día y 1.200 millones de estas personas viven en condiciones de extrema marginación, con menos de US\$1 al día (20). Hacia el año 2005 en América Latina se encontraba un volumen de pobreza e indigencia que ascendía a 213 millones y 88 millones de personas respectivamente (21). Se considera que $66.3 \%$ de la población de Colombia es pobre y $26 \%$ se encuentra en la indigencia (21). La pobreza en la población anciana es una constante en América Latina; en Colombia las personas ancianas constituyen el 35\% de los pobres (21). Se complica la situación porque menos del $20 \%$ de la población anciana está pensionada o con jubilación; es decir, en Latinoamérica aumentan los hogares y personas envejecidas en los grupos socioeconómicos que no disponen de recursos suficientes para garantizar su seguridad económica, en un momento donde el Estado se vuelve cada vez más líquido y la familia es obligada a suplir la esfera que se derrite (19).

Frente a la situación actual, narra Esperanza:

Nosotros tres, o sea mi niña, mi papá y yo estábamos almorzando en el comedor comunitario... aunque a veces no teníamos las moneditas que hay que pagar y la niña se iba al colegio sin almorzar... pero bueno, solo tenía que rebuscar la plata para el desayuno y la comida, pero a un pobre todo le pasa... Un día, me dijeron que solo podía recibir una cosa: o el bono de $\$ 80.000$ o el almuerzo... Hablé con mi papá y decidimos tener el bono... yo sigo esforzándome y hago lo que sea para que no falte nada en esta casa (Esperanza). 
Las familias se ven enfrentadas al desempleo, el subempleo y la informalidad, perciben la disminución del consumo en los rubros de alimentos, la asistencia escolar, el acceso en general a servicios públicos como la salud, la seguridad social y todos aquellos aspectos monetarizados dentro de la sociedad, generando un círculo vicioso, por cuanto los hijos(as) de familias pobres tienen menos oportunidades de acceder a los activos de la sociedad y así continúa la ineficiencia del poder político, el aumento de las desigualdades y condiciones de inequidad (22).

Esperanza dice:

Recuerdo que cuando estuvo hospitalizado, antes de perder la pierna izquierda, permaneció como tres meses en el Hospital San Lucas donde le mandaron un examen que valía $\$ 300.000$ y no lo podiamos pagar y ni lo dejaban salir hasta pagar una deuda de $\$ 150.000$, me tocó poner una tutela al Hospital, casi todos los días estaba pendiente de la respuesta para poder solucionar el problema de salud de mi papá, la gané a los tres meses y fue la única manera para que pudiera salir. En todo ese tiempo no conté con mis hermanos, ninguno me ayudó a pesar de que ellos siempre contaron conmigo cuando tenían a mi papá.

El cuidado ofrecido en el seno de la familia para ayudar a los familiares enfermos, discapacitados, envejecidos o debilitados afecta más a mujeres que a hombres y genera cargas económicas, emocionales y de calidad de vida para las personas que lo asumen. Al volverse líquido el papel de protección del Estado, la familia viene soportando en la esfera privada la dura carga de cuidar, acumulando muchas más desventajas sociales y quedando surcados en un círculo de humillación e indignidad:

La verdad, no comprendía como todo eso había pasado, estuve en el hospital, rodeado de muchos médicos pero por falta de plata para un examen me dejaron dañar mi pierna... me cortaron tan arriba, que tuvieron que rellenarme con unos pitillos, pa' poder darle forma al inicio de la pierna que me quedaba... No volví al sistema de salud ni al médico, cada salida nos cuesta un ojo de la cara, el solo taxi de ida y vuelta cuesta 70 mil pesos... decidimos con Esperanza no volver por el mal trato que dan allá. ¡Ah! Y ¿para qué ir? ya sé cuáles son las pastas que el médico siempre me receta y cada cuanto me las tengo que tomar... (Octavio).

Los Vargas, como muchas otras familias en el mundo glocalizado luchan por mantener la dignidad, son grandes los esfuerzos que hacen para sobreponerse a las adversidades de la vida, de lo cotidiano y del derecho a recibir una atención de salud digna, oportuna y de calidad. Emergen prácticas de cuidado y decisiones que no siempre les favorece pero que les permite seguir viviendo, ante lo cual se requiere protegerlos para que su capacidad de respuesta no se vaya a ver todavia más deteriorada, y de esta manera, poder promover la humanización y dignidad a un gran número de familias que 
demuestran en su cotidianidad silenciosa y privada, el valor, el coraje de la dignidad de involucrarse con el otro, de comprometerse y seguir viviendo.

\section{Conclusiones}

La familia Vargas invita a pensar en el papel interconectado que juegan los profesionales de la salud, como seres en interdependencia con el sufrimiento, las presiones del medio y como fuente de esperanza por la dignidad humana en lo glocal. Nos lleva a asumir una responsabilidad por la inclusión social, por la búsqueda de la ciudadanía y por ser voz pública y privada de la dignidad $(23,24)$.

Se requiere enfrentar las estructuras sociales, las cuales determinan las condiciones materiales de vida que hacen difícil la vida de estos ancianos, donde la dignidad humana se constituye en imperativo moral en lo personal y en lo social, en lo global y local-glocal para comprender cada vez más el mundo de las personas mayores en situación de vejez, discapacidad y pobreza, en congruencia con las politicas públicas específicas para este grupo poblacional longevo y vulnerable, con el fin de garantizar una mejor calidad de vida (25-28).

Se requiere propender por unas politicas públicas más acordes con la cultura, las necesidades percibidas, sentidas y manifiestas de esta población que va en aumento, viviendo en situación de pobreza, vejez y discapacidad. Así como, concientizar a los profesionales de la salud cercanos al sufrimiento de lo humano, tal vez, les resulte posible tomar la bandera de la dignidad humana, construir en la cotidianidad prácticas de cuidado de los humanos y de lo humano, viendo más allá de los órganos, de la enfermedad y lo anormal. Esta fue la mayor riqueza del trabajo con historia oral, pues comenzamos estudiando las dinámicas de cuidado cotidiano hacia los ancianos y se llegó a un universo de lucha por la dignidad con múltiples facetas.

\section{Financiación}

Esta investigación se realizó en la Universidad Nacional de Colombia, Sede Bogotá - Facultad de Enfermería, Grupo de Investigación en Cuidado Cultural de la Salud. Cra 50 No 27 - 70, oficina 702. Bogotá D.C. Colombia. Proyecto 8007032 - Financiado por la Dirección de Investigación Sede Bogotá - DIB.

\section{Conflicto de interés}

Las autoras no tienen conflictos de interés entre investigadores, instituciones financiadoras ni participantes del estudio.

\section{Referencias}

1. Naciones Unidas. La situación demográfica en el mundo, 2014. Informe conciso. ST/ESA/SER.A/354. Cap. VII. Envejecimiento de la población. NuevaYork; 2014. p. 24.

2. OMS, Banco Mundial. Informe mundial sobre la discapacidad. Resumen. Ginebra, Suiza; 2011.

3. Barrantes Monge M. Género, vejez y salud. Acta bioeth. 2006; 12 (2):193-197. ISSN 1726-569X. 
4. Naciones Unidas. Informe de la Segunda Asamblea Mundial sobre el Envejecimiento. Madrid: abril de 2002. [Internet] 2002 [Citado el] Disponible en: http://www.monitoringris.org/documents/norm_glob/ mipaa_spanish.pdf

5. OMS. Envejecimiento activo: un marco político. Rev Esp Geriatr Gerontol. 2002; 37 (S2):74-105. ISSN 0211-139X.

6. DANE 2010. Proyecciones nacionales y departamentales de población 2005-2020. Estudios Poscensales No. 7 Bogotá: departamento Admirativo Nacional de Estadísticas (DANE) Centro Andino de Altos Estudios (CANDENE).

7. Ministerio de Salud y Protección Social. Envejecimiento demográfico. Colombia 1951-2020. Dinámica Demográfica y estructuras poblacionales. Bogotá. 2013.

8. Montenegro A. Historia oral e memoria: a cultura popular revisitada. $3^{a}$ ed. São Paulo: Contexto; 1994.

9. Diccionario Oxford de Nuevas Palabras. Sarah Tulloch (Comp.). Oxford: OUP. Oxford University Press; 1991. p.134.

10. López L. Los saberes culturales en salud en un mundo glocalizado. Index Enfer. 2010; 19(2): 5-7.

11. Bauman Z. Modernidad líquida. México DF: Fondo de Cultura Económica; 2003.

12. Robertson R. (2003). Glocalización: tiempo-espacio y homogeneidadheterogeneidad. En: J. Monedero (Ed.). Cansancio del Leviatán: problemas políticos de la mundialización. Madrid: Trotta, 2003. p. 261-283.

13. Ministerio de Salud. Resolución 8430 de 1993. Por la cual se establecen las normas científicas, técnicas y administrativas para la investigación en salud. Bogotá; 1993.

14. Meihy JC. Manual de história oral. $5^{\mathrm{a}}$ ed. São Paulo: Loyola; 2005.

15. Fuente: Hospital del Sur - ASIS Local-2013. Diagnóstico local con participación social 2012. Localidad de Kennedy Territorios Saludables. Hospital del Sur E.S.E vigencia marzo 2013 - febrero 2014. Bogotá; 2014.

16. Plan de Desarrollo Local de Kennedy 2013-2016 Bogotá Humana Kennedy territorio de oportunidades para ser más humanos.[Internet] 2015 [Consultado septiembre 2015]. Disponible en: http://www.kennedy.gov.co/index.php/desarrollo-local/2013-09-23-23-42-29]

17. Plan de desarrollo económico, social ambiental y de obras públicas 2013- 2016. Localidad de San Cristóbal. [Internet] 2013 [Consultado septiembre 2015]. Disponible en: http://www.sancristobal.gov.co/index.php/desarrollo-local/2013-09-23-23-42-291

18. Cárdenas A, López AL. Analysis matrix of resilience in the face of disability, old age and poverty'. International Journal of Disability, Development and Education. 2010; 57 (2):175-189.

19. Bauman $Z$. Amor líquido. Acerca de la fragilidad de los vínculos humanos. Trad. Rosenberg M y Arrambide J. Buenos Aires: Fondo de Cultura Económica; 2005.p. 8, 48

20. World Bank. World Development Indicators, 2005. Washington DC. World Bank 2005. [Internet] Disponible en: http://wwwwds.worldbank. 
org/external/default/WDSContentServer/WDSP/IB/2005/01/31/0000 09486_20050131102753/Rendered/PDF/31451A.pdf

21. CEPAL (Comisión Económica para América Latina y el Caribe). La Protección social de cara al futuro: acceso, financiamiento y solidaridad. Documento informativo. Santiago de Chile; 2006.

22. CID (Centro de Investigaciones para el Desarrollo). Bien-Estar: Macroeconomía y Pobreza. Informe de Coyuntura 2003. Universidad Nacional de Colombia, Bogotá; 2004.

23. Dalai Lama. La politica de la bondad. Recop y edit. Piburn S. Alicante: Ediciones Dharma; 1994.

24. Bauman Z. La sociedad sitiada. Trad. Rosenberg M y Zaidenwerg E. Buenos Aires: Fondo de Cultura Económica; 2004.

25. Lloyd-Sherlock P, McKee M, Ebrahim S, Gorman M, Greengross S, Prince M, Pruchno R et al. Population ageing and health. Lancet. 2012; 379 (9823):1295-6.

26. León Correa FJ. Pobreza, vulnerabilidad y calidad de vida en América Latina: Rretos para la bioética. Acta bioeth. 2011; 17 (1):19-29. ISSN 1726-569X. [Internet] 2011. Disponible en: http://www.scielo.cl/scielo.php?pid=S1726-569X2011000100003\&script=sci_arttext

27. Reyes Torres I, Castillo Herrera JA. El envejecimiento humano activo y saludable, un reto para el anciano, la familia, la sociedad. Rev Cubana Invest Bioméd 2011; 30 (3):454-459. ISSN 0864-0300.[Internet] 2011. Disponible en:http://scielo.sld.cu/scielo.php?script=sci_arttext\&pid =S0864-03002011000300006

28. AARP, American College of Sports Medicine, American Geriatrics Society, The Centers for Disease Control and Prevention, The National Institute on Aging, The Robert Wood Johnson Foundation. The development of the National Blueprint. Increasing Physical Activity Among Adults Age 50 and Older. Princeton, New Jersey. [Internet] 2001. Disponible en: http://www.activeforlife.info/about_the_program/files/blueprint.pdf 